\title{
Challenge toward breakage of RLS trade-off for EUV lithography by Photosensitized Chemically Amplified Resist ${ }^{\mathrm{TM}}\left(\right.$ PSCAR $\left.^{\mathrm{TM}}\right)$ with flood exposure
}

\author{
Seiji Nagahara ${ }^{1 *}$, Michael Carcasi ${ }^{2}$, Hisashi Nakagawa ${ }^{3}$, Elizabeth Buitrago ${ }^{4}$, Oktay Yildirim ${ }^{5}$, \\ Gosuke Shiraishi ${ }^{6}$, Yuichi Terashita ${ }^{6}$, Yukie Minekawa ${ }^{6}$, Kosuke Yoshihara ${ }^{6}$, Masaru Tomono ${ }^{6}$, \\ Hironori Mizoguchi ${ }^{6}$, Joel Estrella ${ }^{2}$, Tomoki Nagai ${ }^{3}$, Takehiko Naruoka ${ }^{3}$, Satoshi Dei ${ }^{7}$, \\ Masafumi Hori ${ }^{7}$, Akihiro Oshima ${ }^{8,9}$, Michaela Vockenhuber ${ }^{4}$, Yasin Ekinci ${ }^{4}$, Marieke Meeuwissen ${ }^{5}$, \\ Coen Verspaget ${ }^{5}$, Rik Hoefnagels ${ }^{5}$, Gijsbert Rispens ${ }^{5}$, Raymond Maas ${ }^{5}$, Hideo Nakashima ${ }^{1}$, \\ and Seiichi Tagawa ${ }^{8,9}$ \\ ${ }^{1}$ Tokyo Electron Ltd., 3-1 Akasaka 5-chome, Minato-ku, Tokyo 107-6325, Japan, \\ ${ }^{2}$ Tokyo Electron America, Inc., 2400 Grove Boulevard, Austin, TX 78741, USA, \\ ${ }^{3}$ Semiconductor Materials Laboratory, Fine Electronic Research Laboratories, JSR Corporation, 100 \\ Kawajiri-cho, Yokkaichi, Mie, 510-8552, Japan, \\ ${ }^{4}$ Laboratory for Micro-and Nanotechnology, Paul Scherrer Institute, CH-5232 Villigen PSI, \\ Switzerland, \\ ${ }^{5}$ ASML Netherlands B.V., De Run 6501, 5504 DR Veldhoven, The Netherlands \\ ${ }^{6}$ Tokyo Electron Kyushu Ltd.,1-1, Fukuhara, Koshi-shi, Kumamoto 861-1116, Japan, \\ ${ }^{7}$ JSR MICRO NV, Technologielaan 8 - B-3001 Leuven, Belgium, \\ ${ }^{8}$ Graduate School of Engineering, Osaka University, Ibaraki, Osaka 567-0047, Japan, \\ ${ }^{9}$ Institute of Scientific and Industrial Research, Osaka University, Ibaraki, Osaka 567-0047, Japan,
}

\begin{abstract}
This paper proposes a promising approach to break the resolution (R), line-edge-roughness (LER), and sensitivity (S) trade-off (RLS trade-off) relationships that limit the ultimate lithographic performance of standard chemically amplified resists (CAR). This is accomplished in a process that uses a Photosensitized Chemically Amplified Resist ${ }^{\mathrm{TM}}\left(\mathrm{PSCAR}^{\mathrm{TM}}\right)^{* *}$ in combination with a flood-exposure in an in-line track connected to a pattern exposure tool. PSCAR is a modified CAR which contains a photosensitizer precursor (PP) in addition to other standard CAR components such as a protected polymer, a photo acid generator (PAG) and a quencher. In this paper, the PSCAR concept and the required conditions in resist formulation are carefully explained. In the PSCAR process, the sensitivity improvement is accomplished by PAG decomposition to selectively generate more acid at the pattern exposed areas during the flood exposure. The selective photosensitization happens through the excitation of the photosensitizer (PS) generated by the deprotection of the PP at the pattern exposed areas. A higher resist chemical gradient which leads to an improved resolution and lower LER values is also predicted using the PSCAR simulator. In the PSCAR process, the improved chemical gradient can be realized by dual acid quenching steps with the help of increased quencher concentration. Acid quenching first happens simultaneously with acid catalytic PP to PS reactions. As a result, a sharpened PS latent image is created in the PSCAR. This image is subsequently excited by the flood exposure creating additional acid products at the pattern exposed areas only. Much the same as in the standard CAR system, unnecessary acid present in the non-pattern exposed areas can be neutralized by the remaining quencher to therefore produce sharper acid latent images. EUV exposure results down to 15 $\mathrm{nm}$ half pitch (HP) line/space (L/S) patterns using a PSCAR resist indicate that the use of PSCAR has the potential to improve the sensitivity of the system while simultaneously improving the line-width-roughness (LWR) with added quencher and flood exposure doses. In addition, improved across-wafer critical dimension uniformity (CDU) is realized by the use of a PSCAR in combination with a flood exposure using pre $\alpha \mathrm{UV}$ exposure module.
\end{abstract}

Extreme Ultraviolet (EUV) Lithography VII, edited by Eric M. Panning, Kenneth A. Goldberg. Proc. of SPIE Vol. 9776, 977607 - @ 2016 SPIE · CCC code: 0277-786X/16/\$18 · doi: 10.1117/12.2219433 
Keywords: Photosensitized Chemically Amplified Resist ${ }^{\mathrm{TM}}$, PSCAR ${ }^{\mathrm{TM}}$, sensitivity, resolution, LER, RLS trade-off, photosensitizer, photosensitizer precursor, chemical gradient, in-line track, flood exposure, dual acid quenching concept.

*seiji.nagahara@tel.com

** PSCAR ${ }^{\mathrm{TM}}$ and Photosensitized Chemically Amplified Resist ${ }^{\mathrm{TM}}$ are trademarks of Tokyo Electron Ltd. and Osaka University.

\section{INTRODUCTION}

High sensitivity resists are necessary to efficiently reduce the cost of ownership of EUV lithography for its successful insertion into mass production ${ }^{1-5}$. With technology node advances, high resolution and reduced line roughness are increasingly important ${ }^{6-7}$. However, it is challenging to improve the sensitivity, resolution, and LER simultaneously as has been previously shown by many photo-lithographers due to the existence of RLS trade-off relationships among these $\mathrm{s}^{3,7-13}$.

To overcome this challenge, the use of a Photosensitized Chemically Amplified Resist ${ }^{\mathrm{TM}}$ (PSCAR $^{\mathrm{TM}}$ ) was proposed by Tagawa et al. of Osaka University ${ }^{14-15}$. In that publication, the resist demonstrated a sensitivity enhancement of over a factor of nine with $75 \mathrm{~nm}(\mathrm{~L} / \mathrm{S})$ resolution by e-beam lithography in combination with an ultraviolet light (UV) flood exposure $^{14}$.

The use of a PSCAR, in combination with a flood-exposure in an in-line track connected to a pattern exposure tool is proposed in this paper as a promising approach to break the RLS trade-off relationship. The latest PSCAR experimental results are also presented elsewhere ${ }^{16-19}$.

In section 2 of this report, we first discuss the PSCAR concept and required conditions. In section 3, we show the experimental results of EUV resist sensitization realized by PSCAR in combination with a UV flood exposure. Then in section 4, we discuss how to increase chemical gradient by PSCAR in order to improve the LER and resolution via simulation and experiments. Finally in section 5, CDU improvement as realized by the implementation of a pre $\alpha \mathrm{UV}$ flood exposure module is shown.

\section{PSCAR CONCEPT AND REQUIRED CONDITIONS FOR RESIST FORMULATION}

This section discusses the PSCAR concept and required conditions to realize high sensitivity with high resist contrast.

\subsection{PSCAR process flow and expected advantages}

In this section, we will first discuss the main differences between the standard CAR and PSCAR processes.

Figure 1 shows the PSCAR process flow.

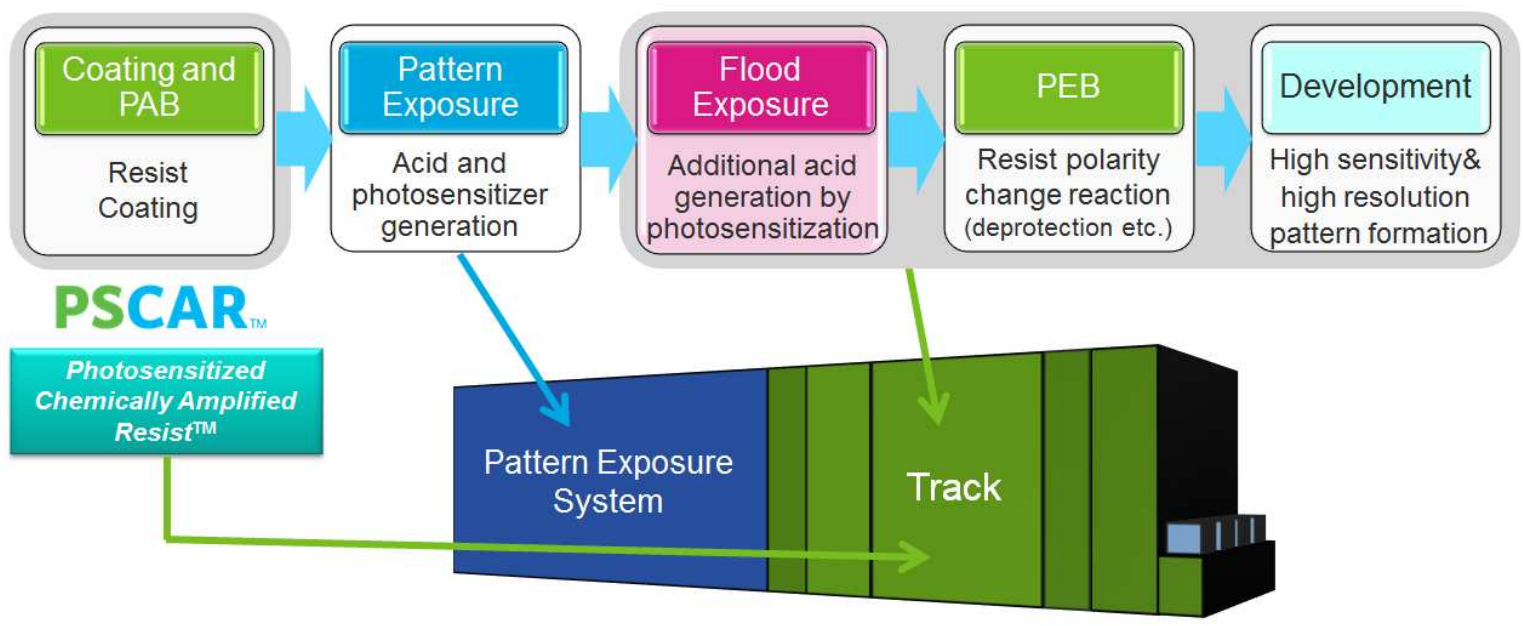

Figure 1. PSCAR process flow. 
The PSCAR is first spin coated onto a wafer in a track system and then transferred to be exposed in an EUV scanner. In the standard CAR system ${ }^{20-22}$, acid is generated during this pattern exposure step. In comparison, in the PSCAR system, a photosensitizer is also generated in addition to an acid product during this pattern exposure step. Then, a UV flood exposure is executed to generate additional acid by photosensitization in the track. Resist-polarity-change reactions happen during the post-exposure bake (PEB) step in the track system (In PEB step, the total acid produced during the pattern exposure and UV-flood exposure steps is used). Finally high sensitivity and high resolution patterns are realized during development.

Figure 2 shows clearly the process flow differences between a standard CAR and PSCAR processes. In the PSCAR process, there is an additional flood exposure step in comparison to the standard CAR process flow. During this flood exposure step, the acid concentration is increased via photosensitizer excitation.
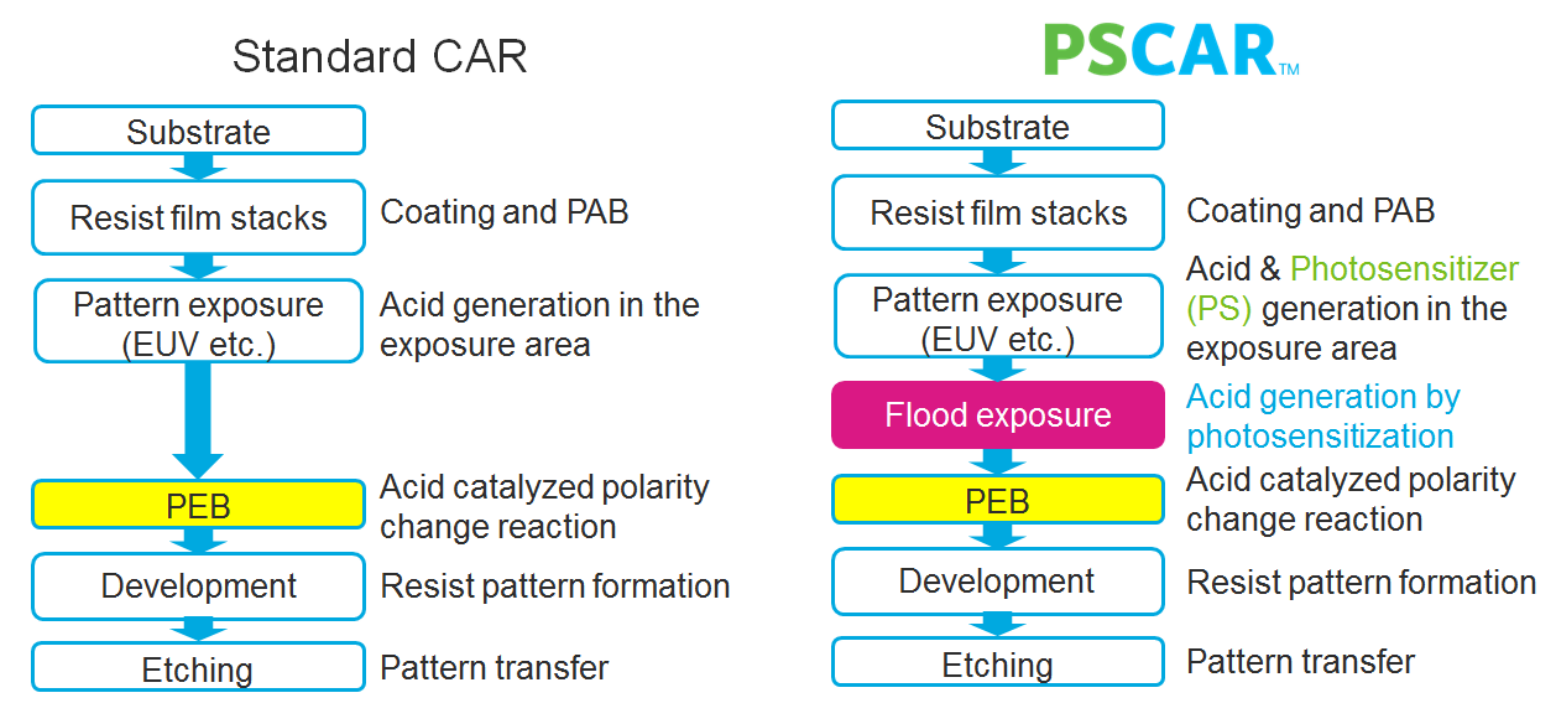

Figure 2. Process steps for standard CAR and PSCAR.

In Figure 3, the initial PSCAR1 (Osaka University sample) demonstration results using an ArF immersion scanner, ASML XT:1900i at Tokyo Electron Kyushu Ltd. are shown.

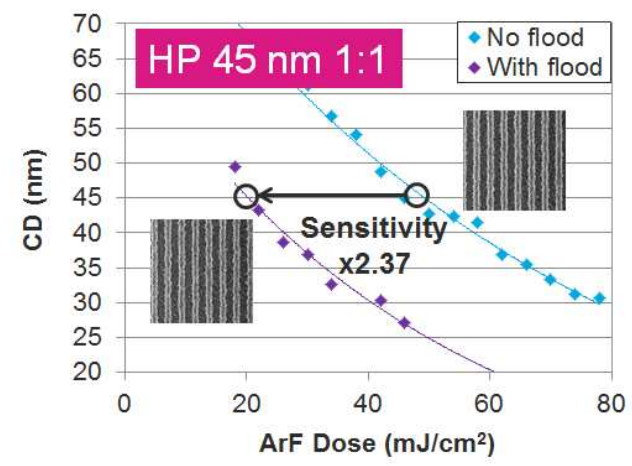

\begin{tabular}{|c|c|c|}
\hline & $\begin{array}{l}\text { Without flood } \\
\text { exposure }\end{array}$ & $\begin{array}{l}\text { With UV flood } \\
\text { exposure }\end{array}$ \\
\hline $\begin{array}{c}\text { Pattern Exposure } \\
\text { Dose }\end{array}$ & $47.3 \mathrm{~mJ} / \mathrm{cm}^{2}$ & $19.9 \mathrm{~mJ} / \mathrm{cm}^{2}$ \\
\hline Sensitivity & $\times 1$ & $\times 2.37$ \\
\hline $\begin{array}{l}\text { Exposure } \\
\text { Latitude (EL) } \\
(\mathrm{CD} \pm 10 \%)\end{array}$ & $20.6 \%$ & $\begin{array}{c}43.0 \% \\
(\times 2.09)\end{array}$ \\
\hline
\end{tabular}

Figure 3. PSCAR1 examined by ArF immersion exposure on ASML NXT:1900i. 
For $45 \mathrm{~nm} \mathrm{~L} / \mathrm{S}$ patterns, more than twice sensitivity and exposure latitude (EL) enhancement were demonstrated using PSCAR1. The results show the potential sensitivity and EL improvement that PSCAR technology might be able to provide when properly optimized.

\subsection{PSCAR reaction steps}

The PSCAR reaction steps are explained in detail in Figure 4. The main difference between a standard CAR and a PSCAR is the addition of PP in the resist formulation.

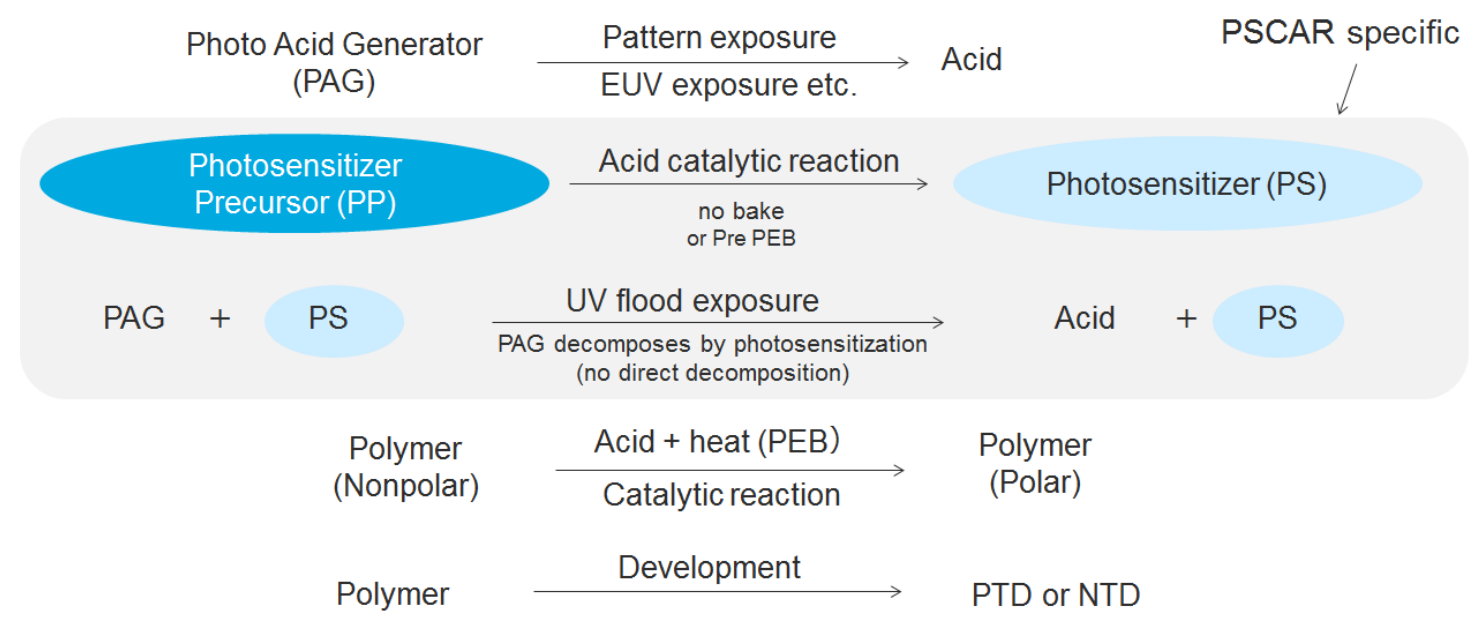

Figure 4. PSCAR reaction steps.

During the pattern exposure step, PAG decomposition is induced leading to the generation of an acid product.

The generated acid catalyzes the PP to PS conversion reaction. Being an acid catalytic chain reaction that can happen at room temperature, the image blur during this step is therefore limited.

Subsequently, additional PAG decomposition happens by photosensitization during the UV flood exposure step in a track system. Direct PAG decomposition cannot happen because the PAG has no absorption in the wavelength $(\lambda)$ region of the flood exposure (see explanation in section 2.3). Therefore, only the PS in the pattern exposed area absorbs the flood exposure wavelengths and generates additional acid in this region alone.

Once the additional acid is generated, the reactions that happen during the PEB are similar to those in standard CAR processes. The total acid is used to switch the polymer solubility by deprotection reactions during PEB. Therefore, the post pattern exposure delay time control until flood exposure and PEB is important in an in-line track system to avoid CD change by acid depletion after pattern exposure.

The subsequent development step is also performed in the track system.

\subsection{PSCAR selective absorption by PS during flood exposure}

Figure 5 illustrates the PSCAR selective absorption by PS during flood exposure. 


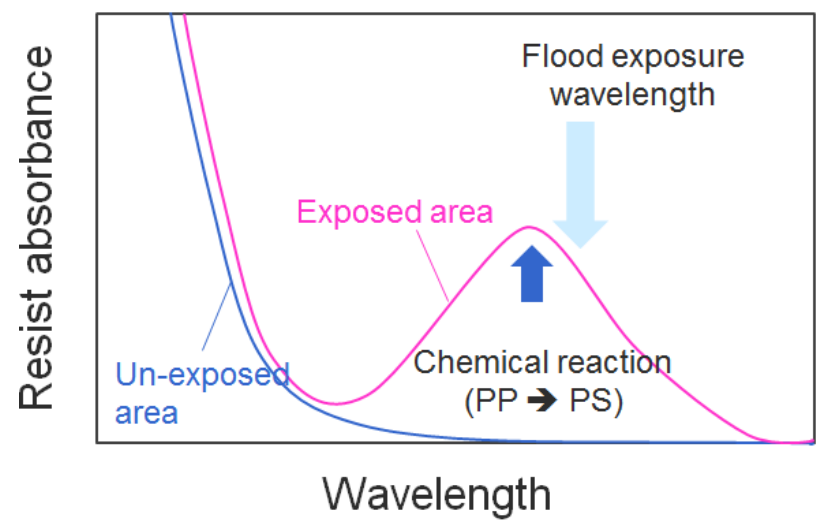

\begin{tabular}{|ccc|}
\multicolumn{4}{c}{ PSCAR Absorption Property } \\
$\begin{array}{c}\text { Resist } \\
\text { Components }\end{array}$ & $\begin{array}{c}\text { EUV Pattern } \\
\text { Exposure }\end{array}$ & $\begin{array}{c}\text { Flood } \\
\text { Exposure }\end{array}$ \\
\hline Polymer & Yes & No \\
\hline PAG & Yes & No \\
Quencher & Yes & No \\
\hline PP & Yes & No \\
PS & Yes & Yes
\end{tabular}

Figure 5. Selective absorption of flood UV light by PS.

The pattern exposure by EUV light $(\lambda=13.5 \mathrm{~nm})$ causes PAG decomposition in the exposed areas ${ }^{20-21}$. The absorption of EUV light by the resist is not very selective because all of the resist components absorb EUV light to a certain extent. The absorbed EUV light generates secondary electrons in the resist. The secondary electrons then react with the PAG molecules to generate an acid product at pattern exposed areas in the resist ${ }^{20-21}$.

On the other hand, the non-pattern exposed resist areas should not have absorption in the flood exposure wavelength. By the conversion of PP into PS with the acid catalytic reactions at the pattern exposed areas, PS only starts to absorb the flood exposure light. With this condition, selective sensitization of PAG can be realized only at the pattern exposed areas.

\subsection{PSCAR PP to PS conversion reaction}

An example of a PP to PS conversion reaction is shown in Figure 6 and is based on the reaction pathway from a ketal/acetal PP to a ketone PS, which is similar to well-known chemistry in conventional acetal resists ${ }^{22}$.

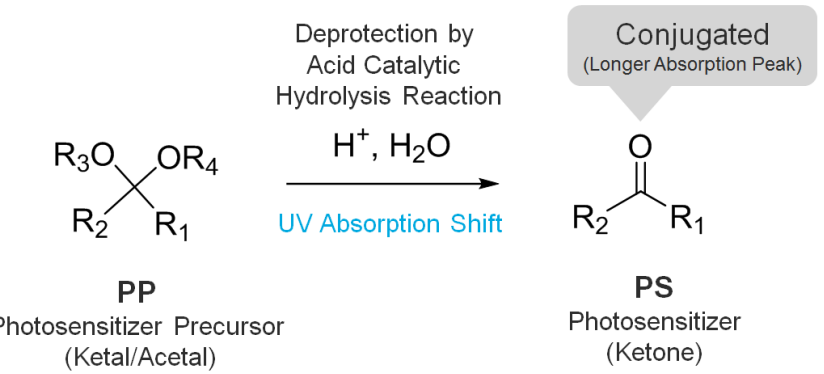

Figure 6. PP to PS conversion reaction (deprotection reaction of protected ketone).

This conversion from PP to PS happens via an acid catalytic reaction. Because of the low activation energy of the acetal/ketal protection group, the deprotection reaction can happen at room temperature. Therefore, the resulting PS image blur will be limited as diffusion length is temperature dependent. It should be noted here that the deprotection reaction of the PP and the acid neutralization reaction by a base quencher are competitive reactions.

By connecting the two aromatic components with a ketone group in the PS through deprotection of PP, the electron conjugation becomes wider in PS in comparison to that of the PP. Therefore, PS UV absorption $\lambda$ can be shifted by $>50$ $\mathrm{nm}$ to longer wavelengths in comparison to that of the PP. This wavelength shift allows for the selective PS absorption of 
light at the flood exposure energy range (see section 2.3). The example of PP and PS spectra and structure of compounds are reported in the other literature ${ }^{17}$.

In Figure 7, the typical acetal/ketal deprotection reaction to generate a photosensitizer is described. This reaction requires acid and atmospheric water ${ }^{23}$. Here it is assumed that the deprotection reaction happens mainly when the wafers are exposed to the atmosphere after the EUV exposure that happens under vacuum.

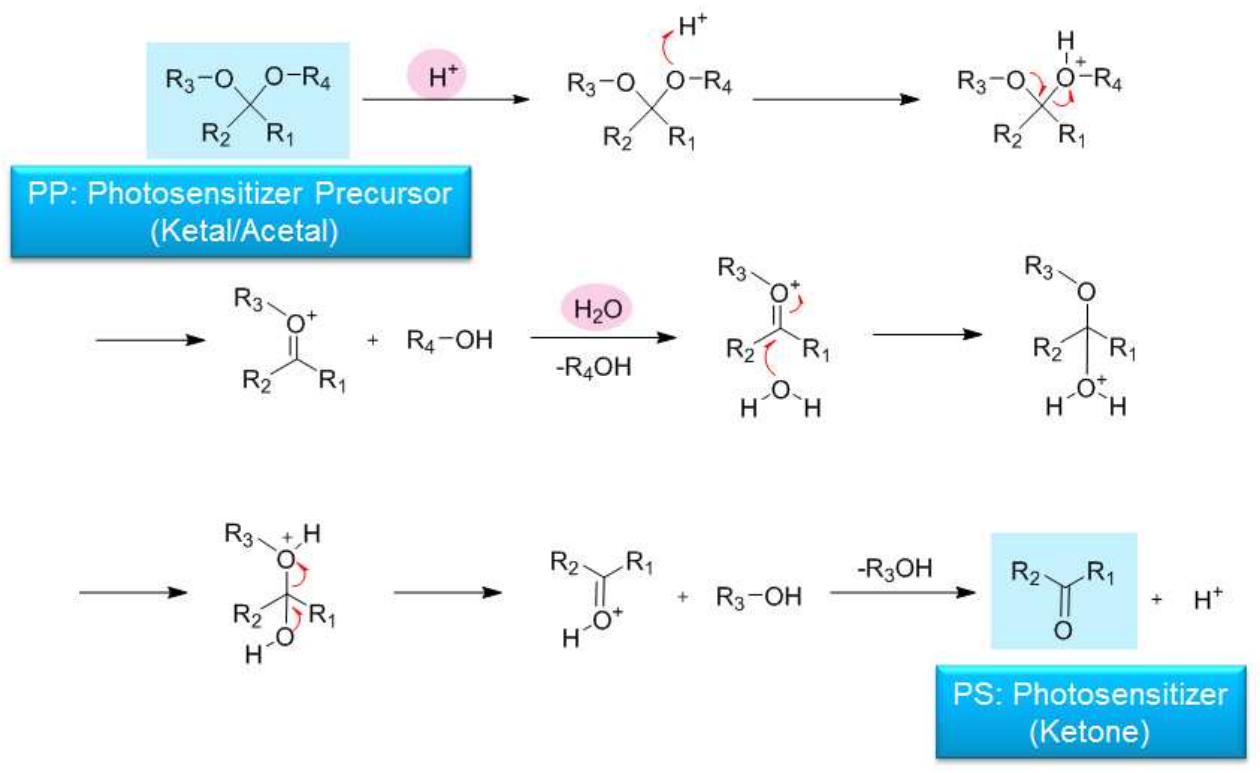

Figure 7. Typical acetal/ketal deprotection to generate photosensitizer.

\subsection{Electron transfer photosensitization of PAG by excited PS during flood exposure step}

Figure 8 illustrates the photosensitization of the PAG through PS excitation during the flood exposure step.

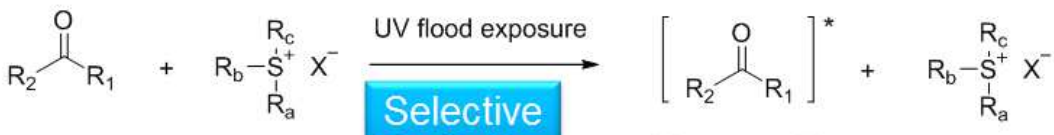

$$
\begin{aligned}
& \text { Photosensitizer (PS) PAG Excitation } \begin{array}{c}
\text { Photosensitizer } \\
\text { excited state (PS }
\end{array} \quad \text { PAG }
\end{aligned}
$$

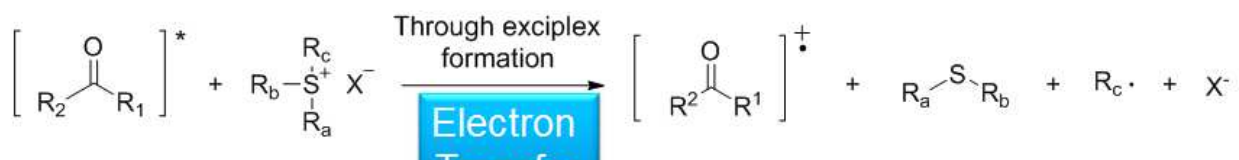

$$
\begin{aligned}
& \text { PS* PAG Transfer Many possible reaction pathways } \\
& \text { of acid generation } \\
& \mathrm{R}^{2} \stackrel{\text { If }}{\mathrm{R}^{1}}+\mathrm{H}^{+} \mathrm{X} \\
& \text { or Derivative Acid }
\end{aligned}
$$

Figure 8. Electron transfer photosensitization of PAG through PS excitation during a flood exposure step.

Photosensitization chemistry is well-known for onium salt based $\mathrm{PAGs}^{24}$. Excitation of the PS can induce acid generation by employing longer flood exposure wavelengths for the photosensitization than the PAG absorption 
wavelength. Photosensitization of onium salt based PAGs is believed to happen mainly by electron transfer from the excited PS to the $\mathrm{PAG}^{24}$. An acid product is then generated by the electron transfer.

The photosensitization reaction should be energetically favorable to be realized as shown in Figure 9.

\section{$\Delta \mathrm{G}=\left(E^{\text {Oxidation }(P S)}-E^{\text {Reduction }(P A G)}\right)-E^{*}$}

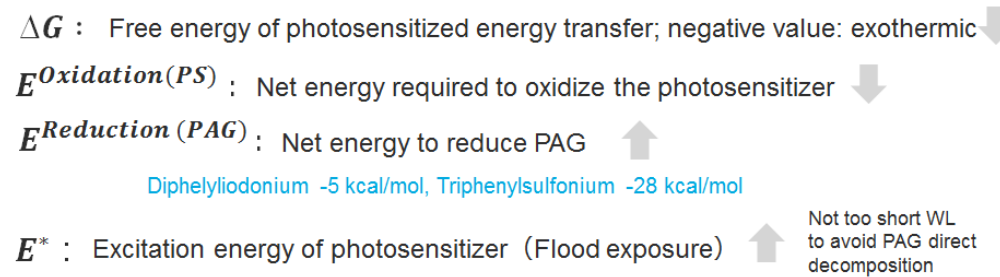

Figure 9. Requirement for Gibbs free energy of photosensitization reaction during flood exposure step ${ }^{24}$.

The Gibbs free energy of the photosensitization reaction (electron transfer) should be negative (exothermic) in order to be thermodynamically favorable as it is shown in Figure 9. Therefore, a lower PS oxidation energy is needed to easily give an electron to a PAG molecule for acid generation. It is also clear that higher PAG reduction energy is favorable to achieve low free energies of electron transfer reaction. The iodonium PAG is well known to have high reduction potential which leads to effective photosensitization ${ }^{24}$. High reduction potential can also be realized with modified sulfonium PAGs ${ }^{24}$.

It has also been found in Figure 9 that the flood exposure wavelength should be short enough to effectively cause electron transfer. However, the wavelength cannot be too short in order to avoid direct photon absorption by the polymer, PAG, quencher and PP during the flood exposures step. The realistic wavelength for the flood exposure is $>350 \mathrm{~nm}$ to avoid side reactions. To get high contrast of the PS against background absorption by the other components in PSCAR, the absorption coefficient of PS at the flood exposure wavelength should be high enough to be effectively excited to generate additional acid.

\subsection{Latent images in standard CAR and PSCAR processes}

Figure 10 shows the general concept of a PSCAR process compared to a standard CAR process in a simplified schematic view ${ }^{14}$.

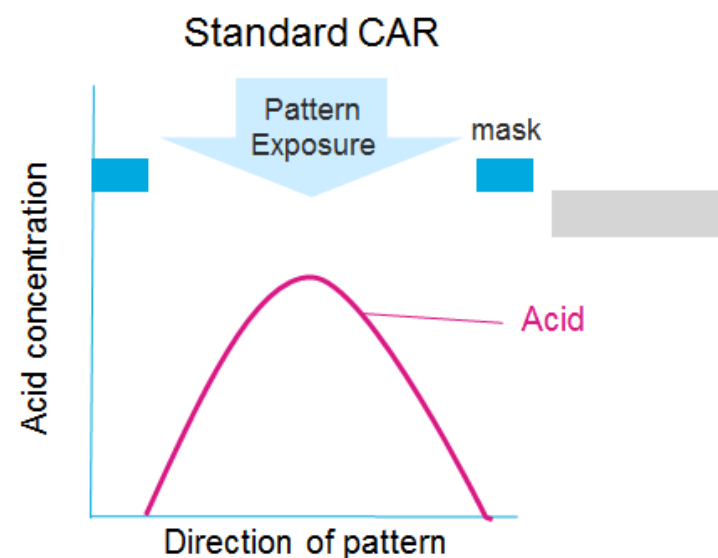

Pattern exposure $\rightarrow$ Acid generation

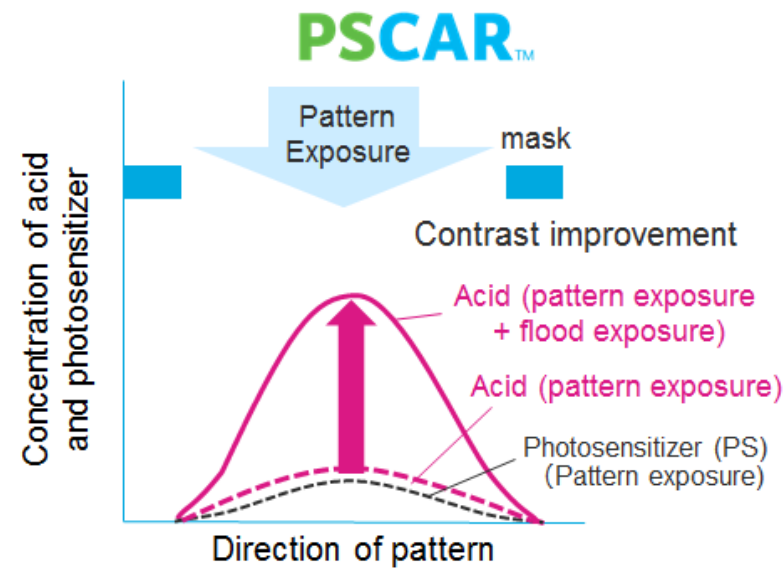

Pattern exposure $\rightarrow$ Acid and PS generation

Flood exposure $\rightarrow$ Additional acid in pattern-exposed area

Figure 10. Latent images in standard CAR and PSCAR processes. 
The produced latent acid image in a standard CAR process after pattern exposure is shown schematically in Figure 10, left side. An acid product is generated during the pattern exposure step and used for polarity switch reactions in PEB.

Similarly, in the case of PSCAR (Figure 10, right side), acid is generated during the pattern exposure step. However, a smaller amount of acid is enough to realize pattern formation compared to a standard CAR. That is because this acid is used to initially catalyze the PP to PS conversion reaction. Photoexcitation of the PS then happens during the flood exposure step creating additional acid in the pattern exposed area only as previously explained. The total amount of acid to be used in the system is the sum of the initial acid produced during the first pattern exposure and the acid generated by the subsequent photosensitization during the flood exposure step. Like this, the total required exposure energy is split between the EUV exposure and the flood exposure to help reduce the required dose for resist patterning by EUV exposure.

Figure 11 illustrates acid image formation in a conventional, non-selective flood exposure to highlight how it differs from the PSCAR process.

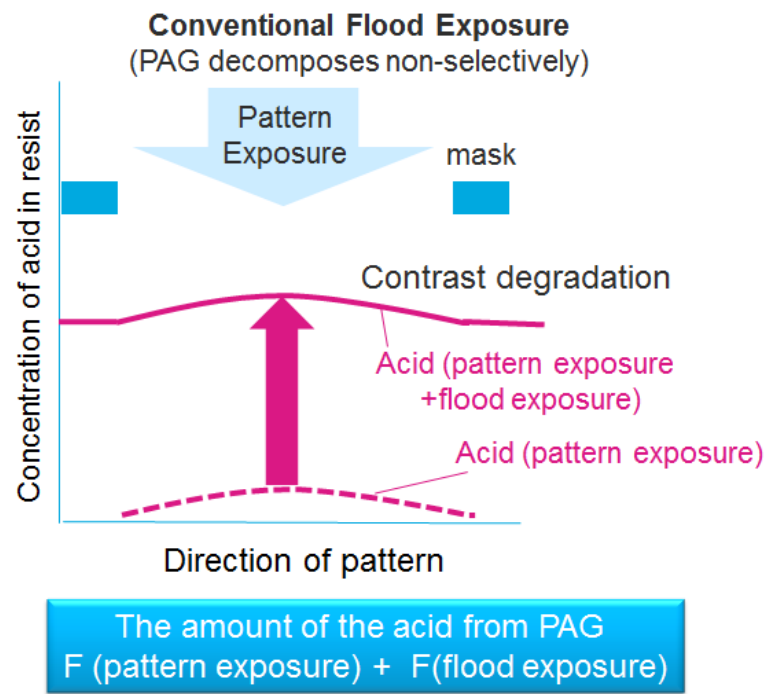

Figure 11. Latent image in a non-selective conventional flood exposure process.

In the conventional non-selective flood exposure, an acid is generated in both exposed and non-exposed areas in different concentrations. The amount of acid generated in the exposed area is roughly equivalent to the total sum of the acid amount generated by the pattern exposure and by flood exposure when the quencher effect is neglected for simplification. This non-selective absorption of flood exposure light causes contrast loss and dissolution of all the resist when too much energy is added during the flood exposure step.

On the other hand, in PSCAR process (Figure 10, right side), only the pattern exposed areas can absorb the flood exposure light. Therefore, acid amount from PAG decomposition is roughly equivalent to the multiplication of pattern exposure and flood exposure if the quencher effect is neglected for simplification.

\section{HIGH EUV SENSITIVITY ENHANCEMENT BY PSCAR}

In this section, the demonstration results of EUV sensitivity enhancement by using PSCAR are shown. The demonstration was done by EUV interference lithography at the Paul Scherrer Institute (PSI) ${ }^{18}$.

Figure 12 shows $18 \mathrm{~nm}$ L/S patterns (25 nm thickness) achieved with PSCAR2 as the UV flood exposure increases. 

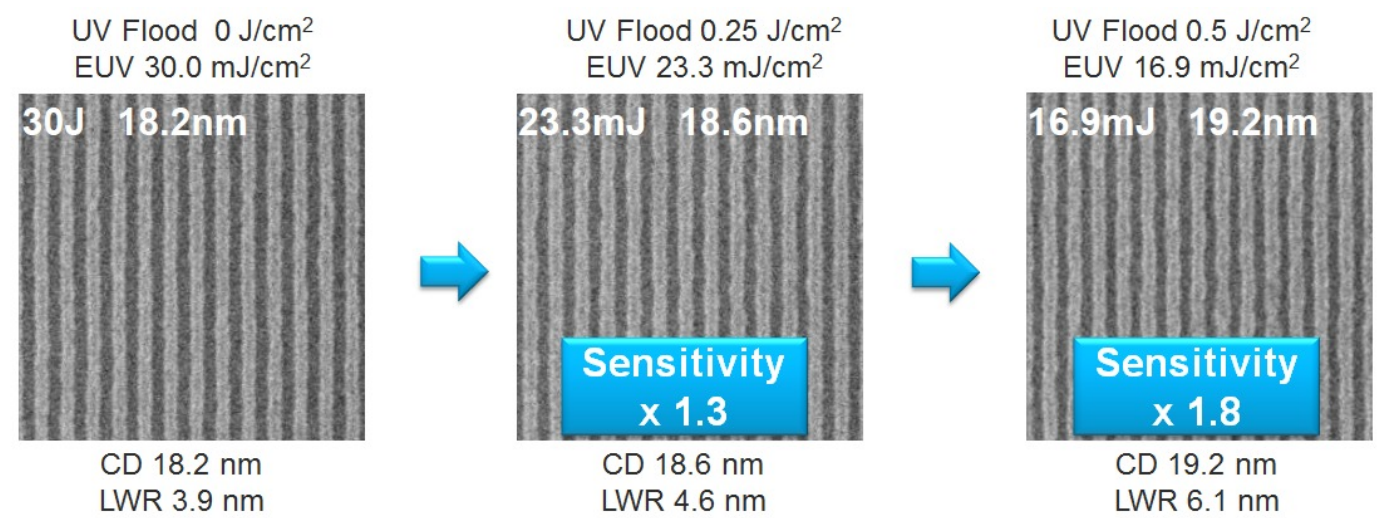

Figure 12. Demonstration of sensitivity enhancement by PSCAR2 with flood exposure. $18 \mathrm{~nm} \mathrm{HP} \mathrm{L/S} \mathrm{patterns} \mathrm{exposed} \mathrm{at}$ PSI $^{18}$.

With a UV flood exposure dose of $0.5 \mathrm{~J} / \mathrm{cm}^{2}(365 \mathrm{~nm})$, the sensitivity increases 1.8 times $\left(16.9 \mathrm{~mJ} / \mathrm{cm}^{2}\right)$ compared to the original EUV sensitivity $\left(30 \mathrm{~mJ} / \mathrm{cm}^{2}\right)$ of the PSCAR. PSCAR fine patterns are therefore found to be resolved with significantly lower dose. However, in this resist, noticeable LWR increase is seen with UV flood exposure dose increase. In the next section, we discuss how pattern quality deterioration can be mitigated.

In Figure 13, the sensitivity enhancement at $22 \mathrm{~nm}$ and $16 \mathrm{~nm} \mathrm{~L} / \mathrm{S}$ resolution from an additional set of experiments is presented.

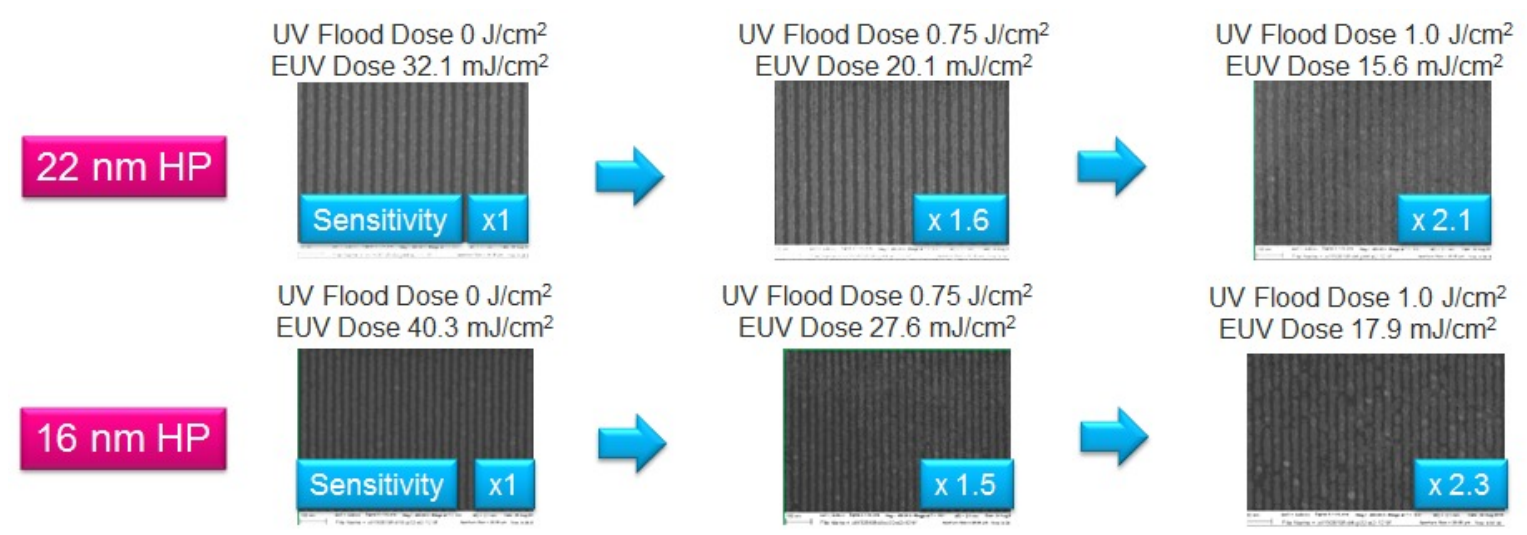

Figure 13. Sensitivity enhancement by PSCAR2 with flood exposure. $22 \mathrm{~nm} \mathrm{HP} \mathrm{L/S} \mathrm{patterns} \mathrm{(upper)} \mathrm{and} 16 \mathrm{~nm} \mathrm{HP} \mathrm{L/S}$ patterns (lower).

EUV pattern dose reduction of over a factor of two is confirmed at 22 and $16 \mathrm{~nm} \mathrm{~L} / \mathrm{S}$. The results clearly show a sensitivity enhancement in EUV lithography is possible by adding the flood exposure step in the PSCAR system. Again, in the very high sensitization condition, the degradation of the pattern shape is seen. This must be improved in the near future for such technology to be commercially successful. Material development and PSCAR learning cycles have just recently started with EUV. Therefore, we expect further improvement in LWR performance with sensitization by improved resist formulation.

\section{CHEMICAL GRADIENT INCREASE BY PSCAR FOR BETTER LER AND HIGHER RESOLUTION}

This section discusses how to increase the chemical gradient aiming for smaller roughness and higher resolution of the resist. 


\subsection{Chemical gradient enhancement by PSCAR for better LER and higher resolution}

How can we face up to the challenge of LER improvement and resolution enhancement with sensitivity enhancement?

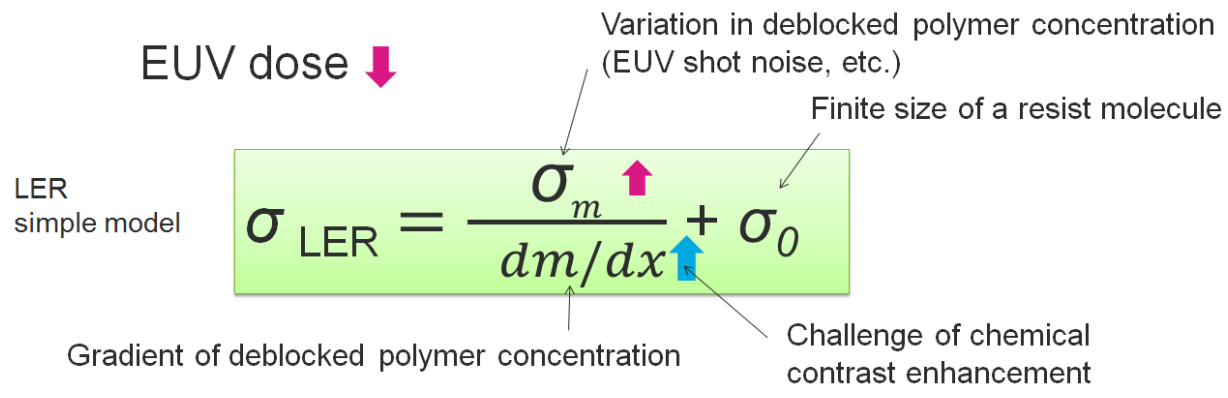

Figure 14. LER improvement challenge by chemical gradient enhancement using PSCAR.

In Figure 14, the LER simple model by Mack is shown ${ }^{25}$. In the case of higher sensitivity with lower EUV dose, the photon shot noise effect becomes more significant. This physical effect cannot be escaped under the fixed condition. PSCAR formulation may also add uncertainty due to the PP and PS distribution. In the case of PSCAR, we would like to tackle the LER by increasing gradient of deblocked polymer concentration. Of course, this approach can be combined with other shot noise mitigation approaches such as increasing the absorption of the resist, increasing the quantum efficiency of acid generation, increasing photoelectron generation efficiency, increasing PAG concentration, etc. ${ }^{26}$

\subsection{LITHOLAB PSCAR lithography simulation model}

To understand the direction of the PSCAR development, we prepared an in-house PSCAR lithography simulator referred to as LITHOLAB.

Figure 15 shows the reaction steps for LITHOLAB PSCAR lithography simulation model.

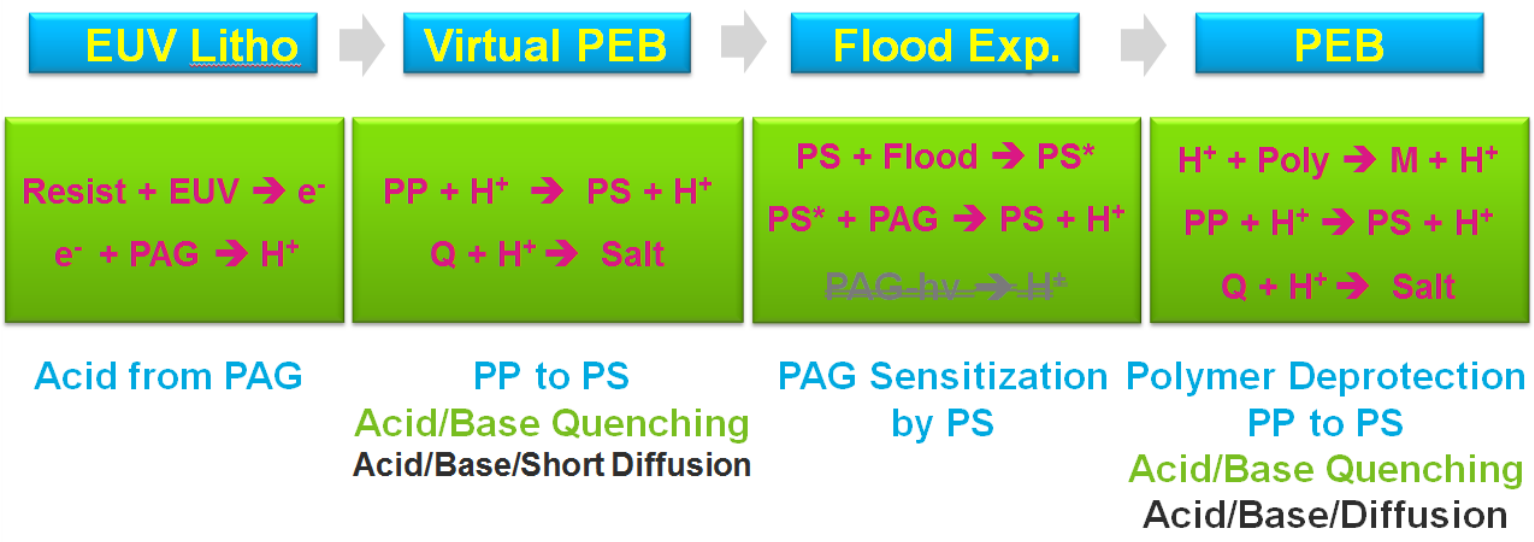

Figure 15. PSCAR reaction steps for LITHOLAB lithography simulator (in-house simulator developed by $\mathrm{TEL}^{\mathrm{TM}}$ ).

For the purposes of this simulation, the acid generation step is assumed to be the same as in a conventional resist model $^{27}$. A virtual PEB step is added to the PSCAR simulation to illustrate reactions between the PP and the acid. Because of the existence of quencher in the system, the neutralization of an acid with a quencher also needs to be considered in this step. The two reactions are competitive reactions at room temperature. Short diffusion is assumed at this step in the simulator. 
Furthermore, excitation of the PS and photosensitization of the PAG during the flood exposure are assumed. The additional acid is generated in the photosensitization step. For simplification, the PAG decomposition by flood exposure is neglected by assuming the PAG absorption at flood exposure wavelength is small enough.

During the PEB, the generated acid catalyzes the deprotection of polymer and PP with acid diffusion. In the simulator, for simplification, the deprotection of PP is not considered to affect solubility of resist in the development step.

In Figure 16, the PSCAR simulation conditions for EUV lithography and simulation examples of resist cross sections are shown.

\section{Illumination and Mask \\ - NA $=0.33$ \\ - Feature $18 \mathrm{~nm} \mathrm{L/S} \mathrm{1:1}$ \\ $-4 \%$ flare is assumed \\ - Dipole X60}

\section{Flood}

- Mid-UV exposure

3. Stack

- Trilayer which is co-optimized for both exposures

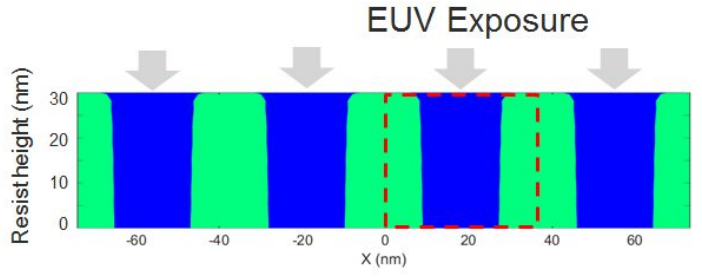

Deprotection during PEB

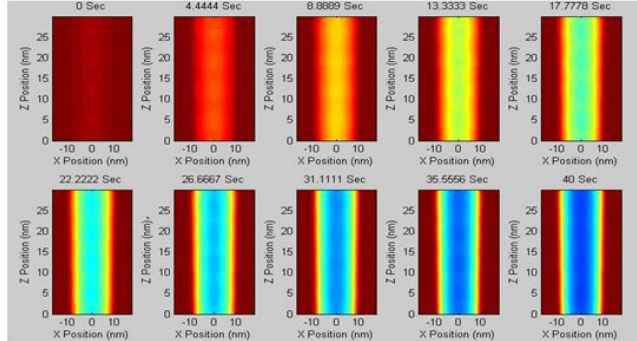

Figure 16. LITHOLAB PSCAR simulation condition and simulation examples of resist cross section.

EUV illumination conditions are set for $18 \mathrm{~nm} \mathrm{1:1} \mathrm{patterns.} 365 \mathrm{~nm}$ UV flood exposure is assumed in the simulation including reflection effects of flood exposure light in the resist. EUV and flood exposure optical simulations are done using PROLITH ${ }^{\mathrm{TM}}$ by KLA Tencor ${ }^{27-28}$. The stack used in this simulation is a trilayer stack which is co-optimized for both pattern and flood exposures by considering the reflection of UV flood exposure and thicknesses for etching hard masks.

\subsection{Simulated latent image in standard CAR and PSCAR}

Figure 17 shows the concentration relationship of acid and quencher in the sequence of process steps for standard CAR (non-PSCAR). 


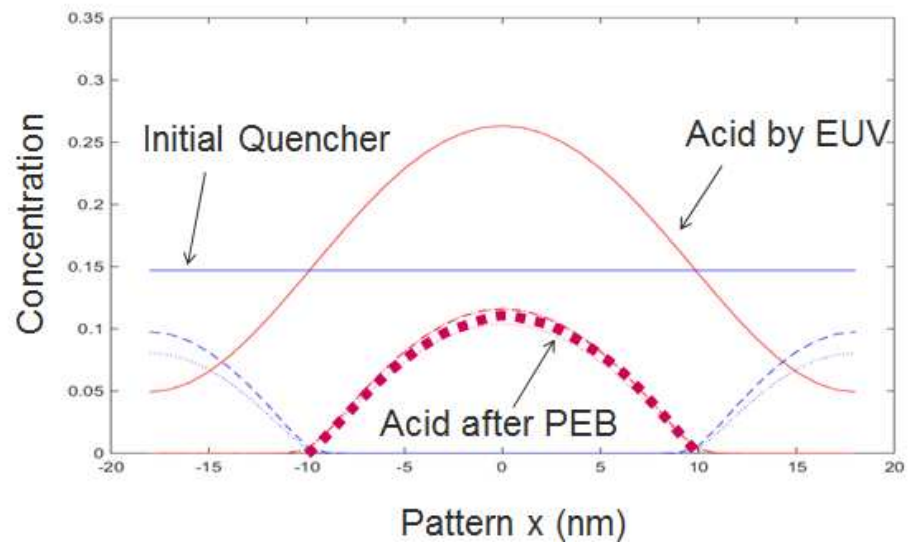

Figure 17. Non PSCAR acid / quencher concentration in a resist.

\section{EUV Dose $21 \mathrm{~mJ} / \mathrm{cm}^{2}$}

\author{
Quencher Loading 0.147 to PAG
}

\author{
Blue: Quencher \\ Red: Acid
}

Solid Line-Post EUV (Q/A)

Dashed Line-Pre Flood (Q/A/PS)

Dots-Post PEB(Q/A)

A quencher loading of 0.147 to PAG and a EUV exposure dose of $21 \mathrm{~mJ} / \mathrm{cm}^{2}$ are assumed to get the right pattern size of $18 \mathrm{~nm}$. The red solid line is acid concentration directly generated by the EUV exposure. After the neutralization by the quencher during PEB, better contrast of acid image is achieved by cutting off the unnecessary acid at the non-exposed areas. In the standard CAR process, this acid amount is directly used for deprotection of the polymer.

Figure 18 shows acid, quencher, and PS concentration in PSCAR.

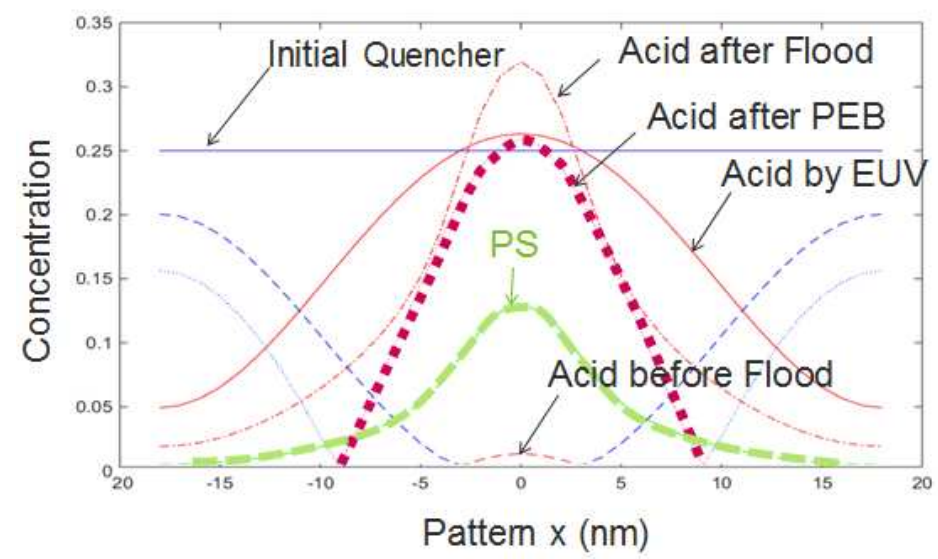

\section{EUV Dose $21 \mathrm{~mJ} / \mathrm{cm}^{2}$}

Quencher Loading 0.25 to PAG

Blue: Quencher

Red: Acid

Green: PS

Solid Line-Post EUV (Q/A)

Dashed Line-Pre Flood (Q/A/PS)

Dash/Dot Line -Post Flood (Pre-PEB) (A)

Dots-Post PEB(Q/A)

Figure 18. PSCAR acid / quencher / PS concentration in a resist.

The same amount of acid is assumed by EUV exposure of $21 \mathrm{~mJ} / \mathrm{cm}^{2}$. In this case, we used a higher quencher amount in PSCAR to adjust the CD at the same EUV dose. Initial acid induces the reaction of PP to PS at room temperature and also the acid can be quenched ( $1^{\text {st }}$ acid quenching). As a result, the acid remaining is very little in this case. However, the PS generated in the resist is enough to generate more acid in the resist. It should be noticed here that the image shape of PS is sharper than original acid image because of the acid quenching before PP to PS conversion. Then after the flood exposure, additional acid is generated and quenched by remaining quencher ( $2^{\text {nd }}$ acid quenching). This "dual acid quenching concept" in PSCAR creates a sharper image than standard CAR. The acid quenching before multiplication by photosensitization is very powerful to enhance resist chemical contrast.

In Figure 19, the comparison of the concentrations of acid with and without PSCAR flood exposure process is shown. 


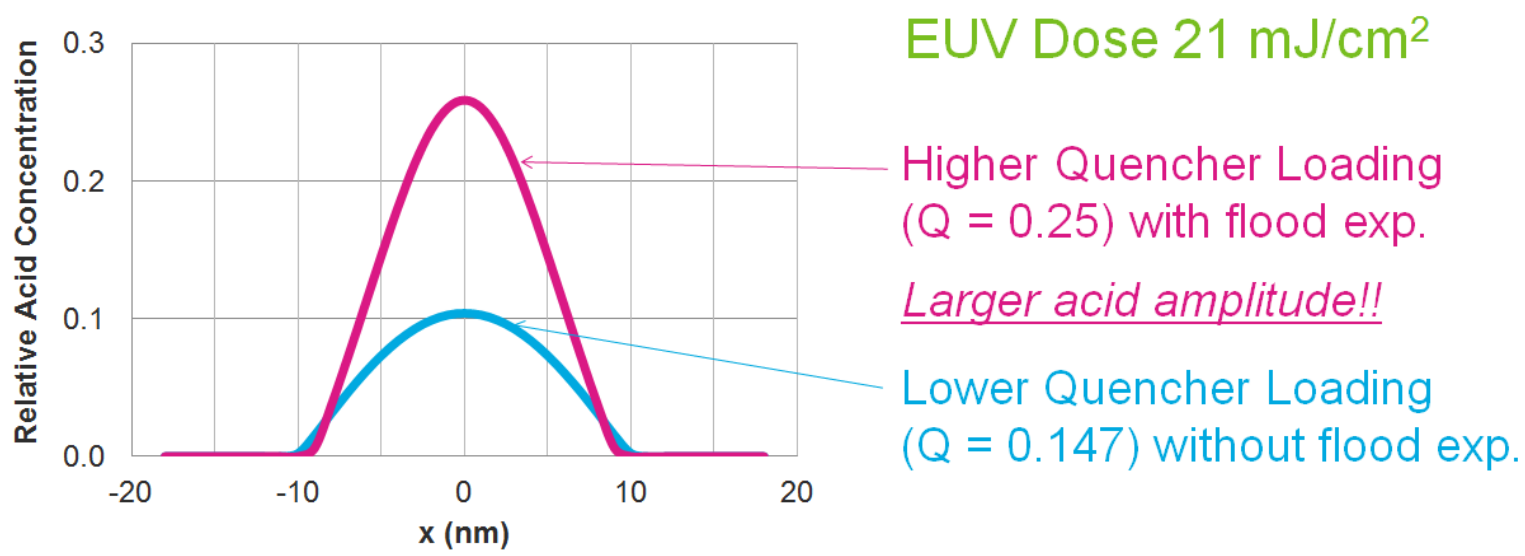

Figure 19. Comparison of acid concentrations after PEB with and without PSCAR flood exposure process.

The EUV exposure dose is the same as the dose in standard CAR in Figure 17 and in PSCAR in Figure 18. The acid amount was set to give the same L/S dimensions. In the case of the same EUV exposure dose as shown in Figure 19, PSCAR can have much larger acid amplitude compared to standard CAR, causing higher acid contrast in the resist.

Figure 20 shows the protected polymer concentration after PEB with and without the PSCAR process.

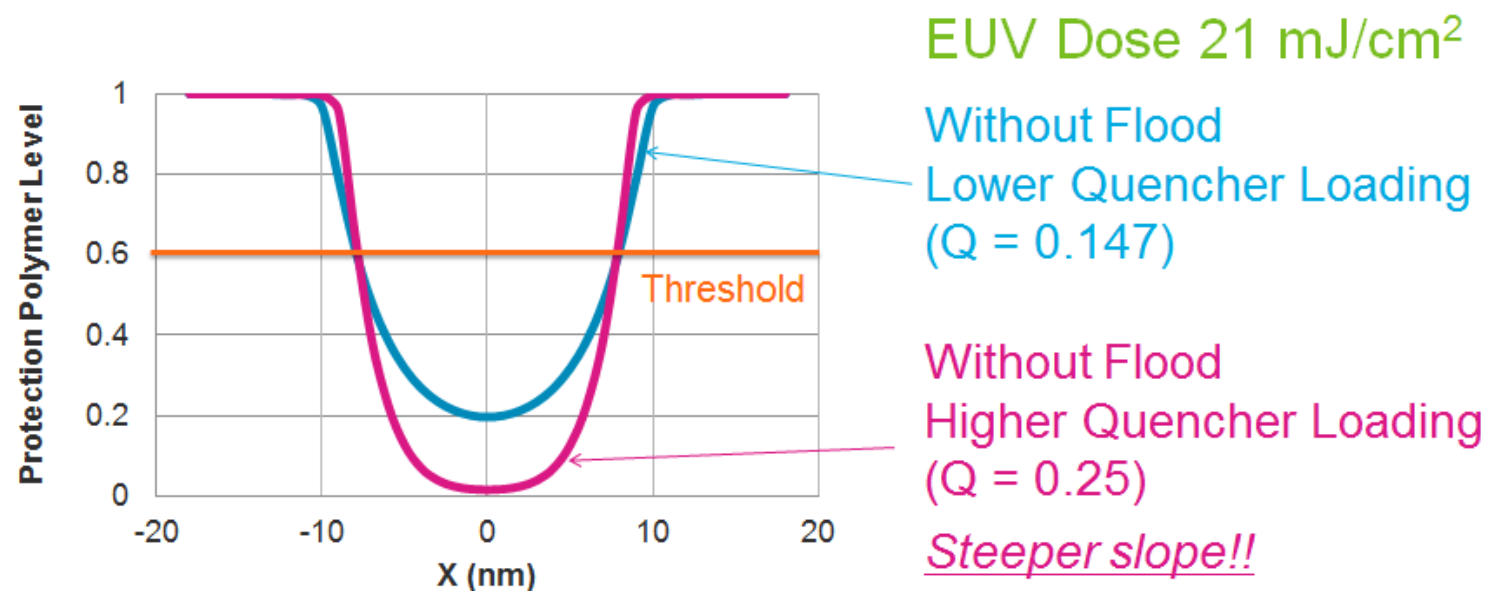

Figure 20. Protected polymer concentration after PEB with and without PSCAR flood exposure process.

As can be seen in Figure 20, the deprotection chemical gradient is much higher for PSCAR with flood exposure at the same EUV exposure dose. This is caused by the higher acid amplitude with higher quencher loading and the dual quenching concept.

With the PSCAR capability of enhancing the resist chemical gradient, we believe that the PSCAR will break the RLS trade-off of standard CAR. This means that PSCAR can realize higher resolution, lower LER due to better contrast, and higher sensitivity by sensitization, when compared to standard CAR.

Figure 21 shows the deprotected polymer gradient for different EUV exposure doses. 


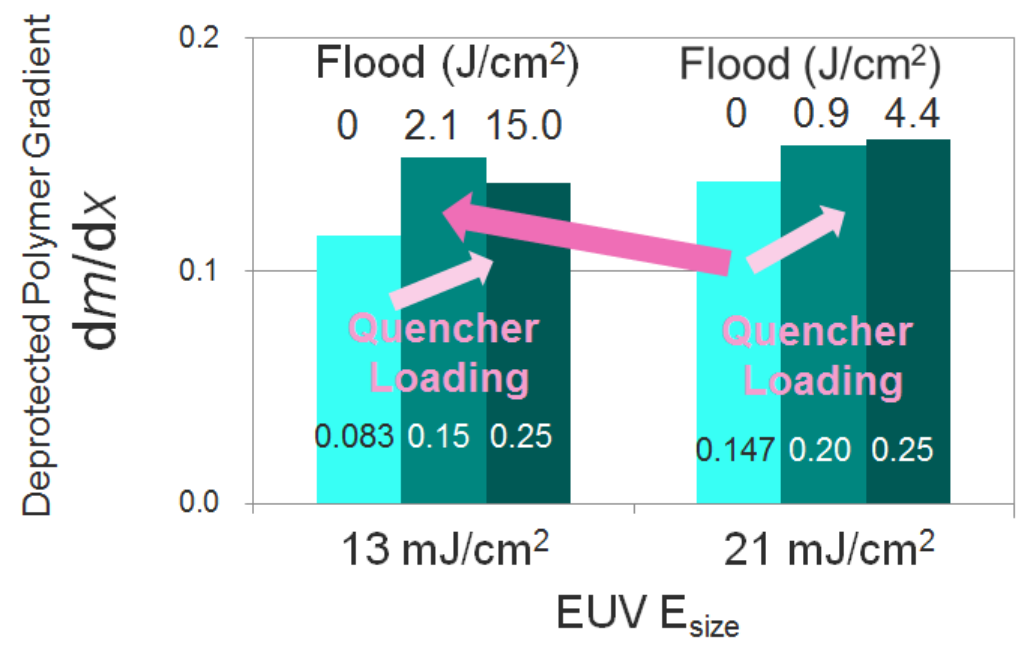

Figure 21. Protected polymer concentration after PEB with and without PSCAR flood exposure process.

In general, PSCAR with flood exposure increases the gradient in the resist at the same doses compared to standard CAR with the help of enhanced amount of quencher and dual quenching steps.

With higher sensitization (lower doses), the chemical gradient obtained at the pattern edge can become smaller with inherently worse LER in the same PSCAR. However, comparing the standard CAR with $21 \mathrm{~mJ} / \mathrm{cm}^{2}$ and the PSCAR at $13 \mathrm{~mJ} / \mathrm{cm}^{2}$, the chemical gradient is higher in PSCAR at $13 \mathrm{~mJ} / \mathrm{cm}^{2}$ than standard CAR at $21 \mathrm{~mJ} / \mathrm{cm}^{2}$. This is again showing the potential of PSCAR to break the standard CAR's RLS trade-off. The effect is more emphasized at very low doses because the acid amount without sensitization becomes too small with the reduction of doses.

As shown above, PSCAR can accommodate more quencher at the same EUV sensitivity with the help of flood exposure. This can lead to higher chemical gradient in the resist by giving the freedom of selection of the best quencher and acid concentration in PSCAR without sacrificing EUV sensitivity. This means quencher addition in PSCAR with flood exposure is one of the solutions to break RLS trade-off of standard CAR.

\subsection{Experimental validation of simulated results}

To examine the assumption in the above simulation, EUV exposure experiments were done by EUV interference lithography at PSI $^{18}$.

Figure 22 shows the results of PSCAR3 at HP $15 \mathrm{~nm} \mathrm{~L} / \mathrm{S}$.

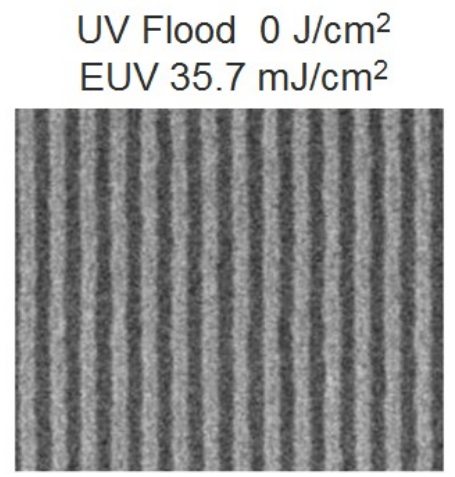

LWR $3.7 \mathrm{~nm}$
UV Flood $0.5 \mathrm{~J} / \mathrm{cm}^{2}$ EUV $34.7 \mathrm{~mJ} / \mathrm{cm}^{2}$

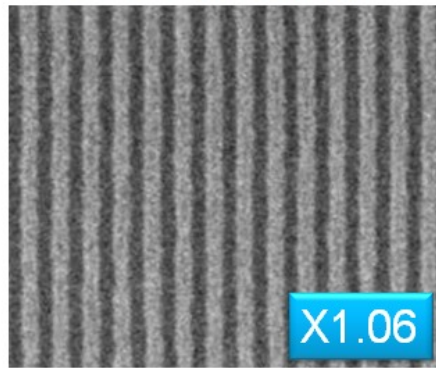

LWR $3.9 \mathrm{~nm}$
UV Flood $1.0 \mathrm{~J} / \mathrm{cm}^{2}$

EUV $31.8 \mathrm{~mJ} / \mathrm{cm}^{2}$

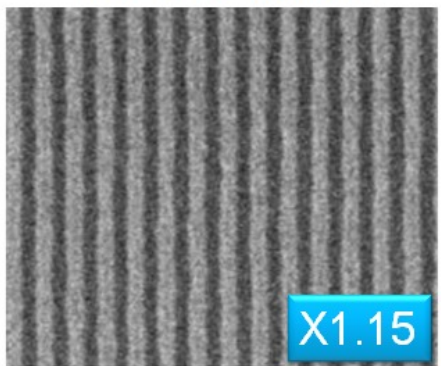

LWR $3.9 \mathrm{~nm}$

Figure 22. $15 \mathrm{~nm} \mathrm{L/S} \mathrm{patterns} \mathrm{made} \mathrm{by} \mathrm{EUV} \mathrm{exposure} \mathrm{on} \mathrm{PSCAR3} \mathrm{with} \mathrm{and} \mathrm{without} \mathrm{flood} \mathrm{exposure} \mathrm{dose.}$ 
With the flood exposure, the sensitivity is enhanced (although the sensitivity enhancement level is lower in the PSCAR3 than in PSCAR2). The LWR values gradually increase with the flood exposure dose in the same resist. This may be partially due to dose reduction effect such as shot noise effect. This tendency can also be partially due to non-selectivity of PP-PS absorption in the current PSCAR. We believe that there is much room for resist improvement in the future, although, as of now, the LER already shows promising values.

In Figure 23, resists with different quencher loading levels are compared.
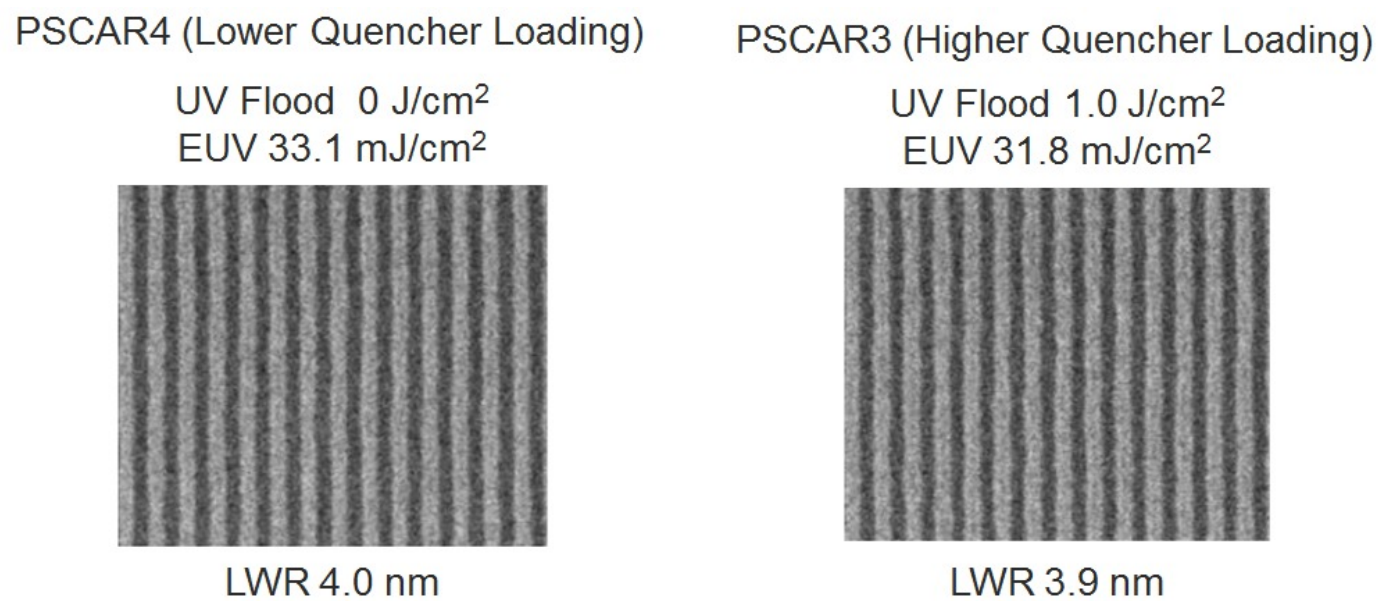

Figure 23. $15 \mathrm{~nm} \mathrm{L/S} \mathrm{patterns} \mathrm{made} \mathrm{by} \mathrm{EUV} \mathrm{exposure} \mathrm{on} \mathrm{PSCAR4} \mathrm{(low} \mathrm{quencher} \mathrm{loading)} \mathrm{without} \mathrm{flood} \mathrm{exposure} \mathrm{and}$ PSCAR3 (high quencher loading) with flood exposure dose.

PSCAR4 has a lower quencher loading and is used without flood exposure. PSCAR3 has a higher quencher loading and is used with flood exposure. Other than quencher loading, the composition of the resists is the same. It is confirmed that with higher quencher loading, the LWR and the EUV sensitivity are improved simultaneously. It is necessary to continue the development of the resists to confirm further clear evidence of the RLS breakage by experiment.

\subsection{Process window change with flood exposure}

Figure 24 shows simulation results of PSCAR as the EUV and flood exposure doses are changed.

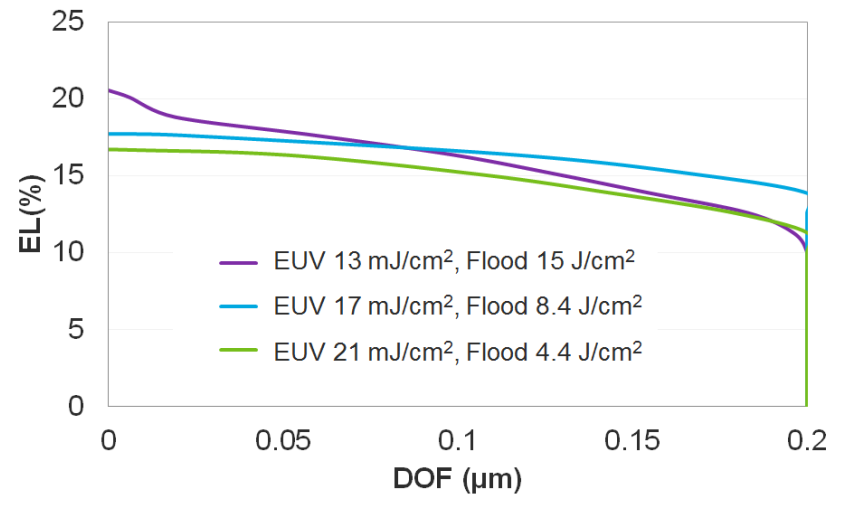

Figure 24. Simulated process window with and without sensitization 
The EUV doses are changed from $21 \mathrm{~mJ} / \mathrm{cm}^{2}$ to $13 \mathrm{~mJ} / \mathrm{cm}^{2}$. The simulated process window (EL and depth of focus, DOF) does not change greatly with sensitivity enhancement in this assumption at $18 \mathrm{~nm} \mathrm{~L} / \mathrm{S}$. However, if we see the graph in detail, noticeable enhancement of EUV pattern exposure latitude with UV flood exposure is observed, as is also seen in ArF immersion experiments in Figure 3.

From the results, it is expected that PSCAR will give more freedom in balancing the resist performances, namely sensitivity level (cost) versus process window.

\section{BETTER CD UNIFORMITY BY PSCAR FLOOD EXPOSURE}

In this section, CDU simulation results after resist photosensitization using TEL's pre $\alpha$ UV flood exposure module are shown.

In Figure 25, the CDU of PSCAR with and without flood exposure are compared using ArF immersion exposure tool (ASML XT:1900i at Tokyo Electron Kyushu Ltd.).

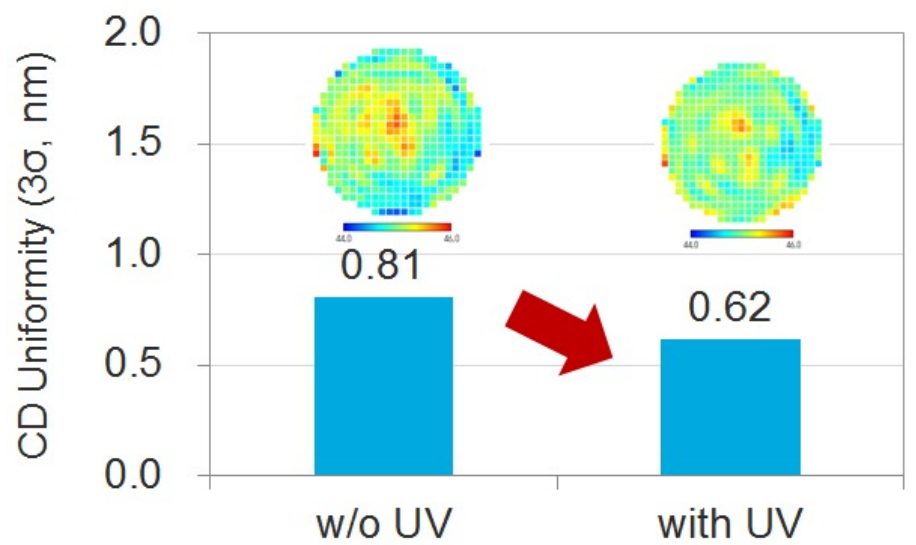

Figure 25. CDU results with and without resist photosensitization using TEL's pre $\alpha$ UV exposure tool. The ArF immersion patterning dose without UV flood exposure is $28 \mathrm{~mJ} / \mathrm{cm}^{2}$ and the dose with UV flood exposure is $18 \mathrm{~mJ} / \mathrm{cm}^{2}$ ( 1.6x sensitivity enhancement condition).

This test is done with PSCAR5 with $\sim 1.6$ times sensitivity enhancement level with $1 \mathrm{~J} / \mathrm{cm}^{2}$ UV flood exposure at $365 \mathrm{~nm}$. $45 \mathrm{~nm} \mathrm{~L} / \mathrm{S}$ patterns are used for CDU evaluation. As can been in Figure 25, the across wafer CDU improves with flood exposure. The $3 \sigma \mathrm{CDU}$ without flood exposure is $0.81 \mathrm{~nm}$ and with flood exposure is reduced to $0.62 \mathrm{~nm}$. The CDU improvement with flood exposure is mainly due to the improved flood dose control. The advantage of the CD control by PSCAR flood exposure is that the flood exposure does not degrade or change the latent image shape greatly in the resist as shown in Figure 21. We believe that the advanced UV flood exposure module capability in a track system will facilitate the PSCAR realization with an advantage of additional CD control knob.

\section{SUMMARY}

PSCAR utilizes a selective photosensitization mechanism by implementing a flood exposure to generate more acid at the pattern exposed areas. With the sensitization, the PSCAR system can enhance the EUV resist sensitivity greatly while still maintaining high resolution. EUV sensitivity enhancement is demonstrated by EUV PSCAR process down to15 nm HP. 
In this paper, a newly developed PSCAR simulation model is used to predict the capability of PSCAR to enhance the chemical gradient by generating more acid in combination with adding more quencher. The dual acid quenching concept which is composed of a first quenching step during the PS formation and a second quenching step during polymer deprotection step is predicted to help enhance the resist chemical gradient according to our simulation results. This gradient enhancement will facilitate the breakage of the RLS trade-off of standard CARs by enhancing resolution and reducing LER.

PSCAR has also been shown to improve the CDU due to the improved dose control in the flood exposure module. This CDU improvement is furthermore realized with sensitivity enhancement. The advantage of the CD control by PSCAR flood exposure is that it does not degrade or change the latent image shape greatly in the resist. This capability can help reduce pattern $\mathrm{CD}$ errors on a wafer.

The addition of the flood exposure step in a track will have a chance to add further possibility of utilizing new chemistry in resists for better performance

\section{ACKNOWLEDGEMENT}

We would like to acknowledge many members of collaboration partners. We also would like to thank Japan Science and Technology Agency (JST) for partial support on this work. Part of this work was performed at Swiss Light Source, Paul Scherrer Institute.

\section{REFERENCES}

[1] Martin van den Brink, "Cost-effective shrink with Holistic Lithography, extended by EUV," EUVL Symposium (2015).

[2] Mark Phillips, Britt Turkot, "EUVL readiness for 7nm," EUVL Symposium (2015).

[3] Jack Chen, "Progress on enabling EUV Lithography for high volume manufacturing," EUVL Symposium (2015).

[4] Arindam Mallik, Julien Ryckaert, Abdelkarim Mercha, Diederik Verkest, Kurt Ronse, Aaron Thean, "Maintaining Moore's law: enabling cost-friendly dimensional scaling," Proc. SPIE, Vol. 9422, 94221N (2015).

[5] Seiji Nagahara, Yusuke Sakurai, Masanori Wakita, Yukio Yamamoto, Seiichi Tagawa, Masanori Komuro, Ei Yano, Shinji Okazaki, "Methods to Improve Radiation Sensitivity of Chemically Amplified Resists by Using Chain Reactions of Acid Generation," Proc. SPIE, Vol. 3999, 386 (2000).

[6] Chris A. Mack, "Line-Edge Roughness and the Impact of Stochastic Processes on Lithography Scaling for Moore's Law," Photonic Innovations and Solutions for Complex Environments and Systems (PISCES) II, Proc., SPIE Vol. 9189, 91890D (2014).

[7] Y. Ma, H. J. Levenson, and T. Wallow, "Line edge roughness impact on critical dimension variation," Proc. SPIE 6518, 651824 (2007).

[8] G. M. Gallatin, "Resist blur and line edge roughness," Proc. SPIE, 5754, 38 (2005).

[9] Gregg M. Gallatin, Patrick Naulleau, Robert Brainard, "Fundamental Limits to EUV Photoresist," Proc. SPIE, Vol. 6519, 651911 (2007).

[10] Robert L. Brainard, Peter Trefonas, Jeroen H. Lammers, Charlotte A. Cutler, Joseph F. Mackevich, Alexander Trefonas, Stewart A. Robertson, "Shot noise, LER and quantum efficiency of EUV photoresists," Proc. SPIE, Vol. 5374, 74 (2004).

[11] Andrew R. Neureuther and C. Grant Willson, "Reduction in $\mathrm{x}$-ray lithography shot noise exposure limit by dissolution phenomena," J. Vac. Sci. Technol. B 6, 167 (1988).

[12] James W. Thackeray, Roger A. Nassar, Robert Brainard, Dario Goldfarb, Thomas Wallow, Yayi Wei, Jeff Mackey, Patrick Naulleau, Bill Pierson, and Harun H. Solak, "Chemically amplified resists resolving $25 \mathrm{~nm}$ 1:1 line-space features with EUV lithography," Proc. SPIE, Vol. 6517, 651719 (2007).

[13] Thomas Wallow, Craig Higgins, Robert Brainard, Karen Petrillo, Warren Montgomery, Chiew-Seng Koay, Greg Denbeaux, Obert Wood, Yayi Wei, "Evaluation of EUV resist materials for use at the $32 \mathrm{~nm}$ half-pitch node,” Proc. SPIE, Vol. 6921, 69211F (2008). 
[14] Seiichi Tagawa, Satoshi Enomoto, Akihiro Oshima, "Super High Sensitivity Enhancement by Photo-Sensitized Chemically Amplified Resist (PS-CAR) Process," J. Photopolymer Science and Technology, Vol. 26, No. 6, 825 (2013).

[15] Seiichi Tagawa, Akihiro Oshima, Satoshi Enomoto, C. Q. Dinh, "High-resist sensitization by pattern and flood combination lithography," Proc. SPIE, Vol. 9048, 90481S (2014).

[16] Seiichi Tagawa, Akihiro Oshima, Cong Que Dinh, Shigehiro Nishijima, "Fundamental aspects of a new process of high-resist sensitization by the combination lithography of EB/EUV pattern exposure with UV flood exposure of photosensitized CAR and non-CAR," Proc. SPIE, Vol. 9799, 9779-78 (to be published) (2016).

[17] Tomoki Nagai, Hisashi Nakagawa, Takehiko Naruoka, Seiichi Tagawa, Akihiro Oshima, Seiji Nagahara, Gosuke Shiraishi, Yukie Minekawa, Yuichi Terashita, Kosuke Yoshihara, Elizabeth Buitrago, Michaela Vockenhuber, Yasin Ekinci, Oktay Yildirim, Marieke Meeuwissen, Rik Hoefnagels, Gijsbert Rispens, Coen Verspaget, Raymond Maas, "Novel high sensitivity EUV photoresist for sub-7nm node," Proc. SPIE, Vol. 9799, 9779-7 (to be published) (2016).

[18] Elizabeth Buitrago, Seiji Nagahara, Oktay Yildirim, Hisashi Nakagawa, Seiichi Tagawa, Marieke Meeuwissen, Tomoki Nagai, Takehiko Naruoka, Coen Verspaget, Rik Hoefnagels, Gijsbert Rispens, Gosuke Shiraishi, Yuichi Terashita, Yukie Minekawa, Kosuke Yoshihara, Akihiro Oshima, Michaela Vockenhuber, Yasin Ekinci, "Sensitivity enhancement of chemically amplified resist and evaluation using EUV interference lithography," Proc. SPIE, Vol. 9776, 9776-36 (to be published) (2016).

[19] Seiichi Tagawa, Akihiro Oshima, Cong Que Dinh, Shigehiro Nishijima, Seiji Nagahara, Michael A. Carcasi, Gosuke Shiraishi, Yuichi Terashita, Yukie Minekawa, Kosuke Yoshihara, Hisashi Nakagawa, Takehiko Naruoka, Tomoki Nagai, "The reaction mechanism and patterning of photosensitized chemically amplified resists," Proc. SPIE, Vol. 9776, 9776-38 (to be published) (2016).

[20] Takahiro Kozawa and Seiichi Tagawa, "Radiation chemistry in chemically amplified resists," Japanese J. Appl. Physics, 49, 030001 (2010).

[21] Seiichi Tagawa, Seiji Nagahara, Toshiyuki Iwamoto, Masanori Wakita, Takahiro Kozawa, Yukio Yamamoto, David Werst, Alexander D. Trifunac, "Radiation and Photochemistry of Onium Salt Acid Generators in Chemically Amplified Resists," Proc. SPIE, Vol. 3999, 204 (2000).

[22] Uzodinma Okoroanyanwu, "Chemistry and Lithography," John Wiley \& Sons.

[23] Sean D. Burns, David R. Medeiros, Heather F. Johnson, Gregory M. Wallraff, William D. Hinsberg, C. Grant Willson, "Effect of humidity on deprotection kinetics in chemically amplified resists," Proc. SPIE, 4690, 321 (2002).

[24] J. V. Crevello, "Cationic polymerization - Iodonium and sulfonium salt photoinitiators," Vol. 62 of the series Advances in Polymer Science, 1 (1984).

[25] Chris A. Mack, "A Simple Model of Line-Edge Roughness," Future Fab International, Vol. 34 (2010).

[26] James W. Thackeray, James F. Cameron, Michael Wagner, Jin Wuk Sung, Warren Montgomery, "Optimization of Low Diffusion EUV Resist on Different Substrates," EUVL Symposium (2011).

[27] Chris A. Mack, "Inside PROLITH: A Comprehensive Guide to Optical Lithography Simulation," Finle Technologies (1997).

[28] PROLITH ${ }^{\mathrm{TM}}$, http://www.kla-tencor.com/Lithography-Software/chip-prolith.html. 